\title{
The first results of the "bevolkingsonderzoek" in the Zaanstreek region in The Netherlands
}

\author{
R. J. L. F. Loffeld ${ }^{1}$ • P. E. P. Dekkers ${ }^{1} \cdot$ B. Liberov ${ }^{1}$ \\ Accepted: 29 April 2018 / Published online: 26 May 2018 \\ (C) Springer-Verlag GmbH Germany, part of Springer Nature 2018
}

\begin{abstract}
This short communication describes the results with respect to stage of colorectal cancer in people detected via screening and patients with clinical complaints.
\end{abstract}

Keywords Colorectal cancer $\cdot$ Colonoscopy $\cdot$ Screening

The "bevolkingsonderzoek" for colorectal cancer (BVO) in The Netherlands was initiated in the expectation that cancer would be detected in an earlier stage, thereby increasing overall survival and disease-free survival. Moreover, the detection and removal of pre-cancerous lesions will help to prevent colorectal cancer. The program is too "young" to do any predictions regarding survival. However, it is possible to investigate the yield of the BVO colonoscopies and to assess the stage of the detected and removed cancers.

For this reason, all colonoscopies in the years 2016 and 2017 in the Zaans Medisch Centrum were evaluated. Patients were sent via the screenings organization but also via their general practitioner because some individuals wanted to have the procedure done in their "own" hospital. The yield of BVO colonoscopies with respect to polyp(s) was high, 81.6 and $79.7 \%$ respectively. No polyp(s) or cancer was seen in 14.6 and $16.6 \%$ of the screenies. With respect to colorectal cancer, patients were divided in two groups. Group 1(control group), patients who underwent normal diagnostic colonoscopy for all kinds of clinical reasons and, group 2, (asymp- tomatic?) people with a positive fecal occult blood test (FOBT) via BVO screening. In group 1, a total of 112 cancers were detected, in group 2 (the BVO group) 29. Statistics were done with chi-square test. There was no difference in age, localization of the tumor, or presence of metastases at presentation. There was no difference in proximal or distal localization of the tumor. In the BVO group, all patients but one underwent surgery. In group 1, 16 patients did not undergo surgery because of the presence of metastatic disease; hence, they received palliative treatment.

Patients in group 2 had a significantly lower T-stage (T1 and T2 46 versus $22 \%, p<0.001)$. There was no difference with respect to the $\mathrm{N}$-stage (N1 and $\mathrm{N} 235$ versus $45 \%, p=$ ns). Despite the fact that this is a small group, these findings are promising. Whether this will ultimately lead to a better overall and disease-free survival (of course with the exclusion of lead time bias) will become clear in the future. With respect to the detection and removal of many polyp(s), it can be expected that the incidence of colorectal cancer will decrease in the near future.

R. J. L. F. Loffeld

loffeld.r@zaansmc.nl

1 Department of Internal Medicine and Gastroenterology, Zaans Medisch Centrum, Zaandam, The Netherlands 